\title{
A New Type-2 Fuzzy Algorithm for Unmanned Aerial Vehicle Image Segmentation
}

\author{
Tingyu Zhong ${ }^{1}$, Wenping Liu ${ }^{*}$, Youqing Luo $^{2}$ and Chih-Cheng Hung ${ }^{3}$ \\ ${ }^{1}$ Institute of Information, Beijing Forestry University, Beijing 100083, China \\ ${ }^{2}$ Institute of Forestry, Beijing Forestry University, Beijing 100083, China \\ ${ }^{3}$ Center for Computer Vision and Security Research, Kennesaw State University, \\ GA 30006, USA

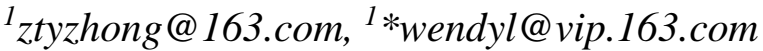

\begin{abstract}
This paper proposes a new type-2 fuzzy algorithm for the Unmanned Aerial Vehicle $(U A V)$ image segmentation. In this algorithm, a logarithmic function is applied to transforming the membership of each pixel which is derived from the Fuzzy C-Means (FCM) algorithm; In other words, this logarithmic function defines a new membership function which can update the cluster centers effectively. The proposed method is called the Logarithmic Functional Fuzzy C-Means Algorithm (LF-FCM) used for the UAV image segmentation. The proposed method achieves better segmentation results than those of the K-means, FCM clustering algorithms and possibilistic c-means algorithm $(P C M)$ in our comparative study for UAV images. A numerical example is provided to exam the behavior and effectiveness of all the algorithms tested. Experimental results demonstrate that the proposed LF-FCM algorithm is efficient and robust for natural UAV images. The Davies-Bouldin $(D B)$ index, the rate of misclassification $\left(P_{\text {error }}\right)$ and the Relative Ultimate Measurement Accuracy (RUMA) are used as quantitative measurement for the comparison.
\end{abstract}

Keywords: Image segmentation; type-2 fuzzy; logarithmic function; fuzzy c-means algorithm

\section{Introduction}

Clustering algorithms are useful in many different applications such as image segmentation, data mining and pattern recognition. It is the process of classifying a set of physical or abstract objects according to a certain similarity criterion. The objective of clustering is to make the within-class samples as similar as possible, and the inter-class samples as different as possible. Fuzzy clustering is one of important fuzzy methods for image segmentation. The most widely used method for image segmentation is the Fuzzy C-Means (FCM) clustering algorithm [1]. The FCM algorithm is achieved by iteratively optimizing the objective function that is dependent on the distance of the pixels to the cluster centers in the feature domain. The FCM algorithm can achieve good image segmentation only for the images without noise or with low noise level. Hence, researchers have proposed different solutions trying to eliminate these problems. Hossein Yousefi [2] applied improved Fuzzy C-Means with spatial information for left ventricular wall segmentation, which can utilized neighboring pixels information to suppress noise and artifact effect. Feng [3] embedded a bias correction into FCM. Then, a non-locally spatio-temporal regularization was used in their algorithm. Tang [4] proposed a double weighted fuzzy clustering method for color image segmentation, using the window-based point density weighted method to calculate the membership matrix and the reliefF algorithm to assign weights to the components of a true color image. Wang [5] presented a novel FCM image segmentation scheme by utilizing local contextual information and 
the high inter-pixel correlation inherent. Zhou [6] carried out region merging according to the region area and the Bhattacharyya distance of two adjacent regions' histogram using the results obtained from the FCM algorithm. Zhu [7] introduced the concept of the neighborhood density to initialize the cluster center to avoid the improper initial value, which is based on the neighborhood correlation of the data space. Guo [8] added spatial penalty terms in the FCM algorithm. These improved algorithms take the spatial information into the consideration in the FCM. All of these algorithms with the weighted membership function improve the performance compared with that of the traditional FCM algorithm. These modified FCM algorithms which are still in the category of type-1 fuzzy technique are adding more features for training in order to have a better performance.

The FCM algorithm is one of the type-1 fuzzy algorithms, and the type-1 method has been used in image segmentation successfully. However, type-1 fuzzy set may have limited capabilities to handle complicated fuzzy conditions, and the type- 2 fuzzy logic provide an alternative to solve the difficulty such as those noisy points using the traditional type-1 fuzzy logic algorithm. Long and Pham [9] used the interval type-2 fuzzy subtractive clustering algorithm, which is based on the extension of subtractive clustering algorithm with fuzzy parameters for image segmentation. Nghiem [10] presented an image thresholding method based on the intuitionistic type-2 fuzzy set method for image segmentation problems. Besides, the intuitionistic type-2 fuzzy set has been formed as an extension of intuitionistic fuzzy set for handling uncertainty. Ouarda [11] proposed a new thresholding method for image segmentation using type-2 fuzzy 2-partition entropy, and type-2 fuzzy sets represent fuzzy sets with fuzzy membership values. Shi [12] used the interval type-2 fuzzy approach into the framework of active contour model. In his approach, both spatial and gray-scale constrains are also taken into consideration when calculating the membership grade. Pham [13] proposed a novel algorithm which combined by the fuzzy co-clustering approach and interval type- 2 fuzzy sets to segment the UC Berkeley image data-sets. Their experimental results showed that the clustering algorithm based on the type-2 fuzzy theory gives better image segmentation. The research has shown that image segmentation based on the type-2 fuzzy logic is more efficient to deal with the problem of image fuzziness. Therefore, a novel type-2 fuzzy logic approach is proposed by transforming the membership function defined from the FCM.

In short, the theory of type- 2 fuzzy logic has some advantages over the type-1 fuzzy set [14]. Based on this motivation, we propose an algorithm called Logarithmic Functional Fuzzy C-Means Algorithm (LF-FCM). In the LF-FCM algorithm, a natural logarithmic function is applied to the membership of each pixel which is derived by using the FCM to transform the membership value. Through this transformation, the LF-FCM will form a new membership function in the clustering procedure. The LF-FCM algorithm enlarges the large membership and decreases the small membership for increasing their difference in order to obtain more accurate segmentation results. This modification in the LF-FCM will contribute more precisely to the update of cluster centers.

The rest of the paper is organized as follows. Section 2 briefly describes the related FCM algorithm. Section 3 sketches the proposed LF-FCM algorithm in detail. Section 4 presents the experimental results on some simple numerical data and UAV images. Section 5 gives the conclusion of proposed algorithm and improvement for future work.

\section{The Fuzzy C-Means Algorithm}

Given a data set $X$ containing $n$ data points, which is expressed as $X=x_{k}(\mathrm{k}=0,1, \ldots, \mathrm{n}-1)$. The clustering problem is to divide the $R=\left\{\mathrm{x}_{0}, \mathrm{x}_{1}, \ldots, \mathrm{x}_{\mathrm{n}-1}\right\}$ into a subset of $s(2 \leq s \leq n)$, which requires the within-class points as similar as possible. The FCM algorithm is to minimize the weighted objective function $J$, which is given below: 


$$
J=\sum_{i=1}^{s} \sum_{k=1}^{n} u_{i k}^{m} d_{i k}^{2}
$$

where $m \in(1,+\infty)$ is a weighting exponent, ${ }^{i k}$ is the degree of membership that the $k^{\text {th }}$ point belongs to the $i^{\text {th }}$ cluster, $s$ is the number of clusters, $d_{i k}^{2}$ is a distance measure between ${ }^{x_{k}}$ and center ${ }^{c_{i}}$ of a fuzzy cluster. The fuzzy cluster center ${ }^{c_{i}}$ and the membership grade $u_{i k}$ are updated using Eqs. (2) and (3) [15].

$$
\begin{array}{r}
u_{i k}=\frac{1}{\sum_{j=0}^{s-1}\left(\frac{d_{i k}}{d_{i j}}\right)^{\frac{2}{m-1}}} \\
c_{i}=\frac{\sum_{k=0}^{n-1} u_{i k}^{m} x_{k}}{\sum_{k=0}^{n-1} u_{i k}^{m}}
\end{array}
$$

The FCM algorithm is sensitive for isolated points and heavy noise signals, so the better results of image segmentation can be achieved only for the images without noise or with low noise level. Many researchers have proposed different solutions trying to eliminate these problems. The proposed algorithm in the study has a better performance for heavy noise images than the traditional FCM algorithm.

\section{The Proposed Logarithmic Functional Fuzzy C-Means Algorithm (LF-FCM)}

The proposed algorithm is a new fuzzy clustering algorithm motivated from the type-2 fuzzy theory. The foundation of LF-FCM algorithm is that the higher membership a pixel has, the probability belonging to that cluster should be high. Simultaneously, it will be more reliable on the update of the cluster centers than the other pixels which have a lower membership.

In the FCM algorithm, the membership function shows the possibility of a pixel belonging to a cluster, which is calculated through the distance between the pixel and the cluster center. However, when a cluster center is updated, it may be imprecise merely taking the membership grade derived from the FCM. Therefore, this paper proposed a function, the natural logarithm function, which will transform each membership value of each pixel, calculated from the FCM. The logarithm function transforms a high (low) membership value into a higher (lower) membership value. This idea is mainly based on the type-2 fuzzy theory. If a membership of a pattern is higher, it will give a more contribution in determining a cluster center. Similarly, if a membership value of a pattern is low, it will give less influence in determining a cluster center. The function abides the following rules: the trend of type- 2 membership degree is consistent with the membership function of FCM, and when the FCM membership value is 0 or 1 , the limit of function is also 0 or 1. The role of the logarithm function is as follows: if the membership value of a pixel is less than a threshold, then its membership value will be appropriately reduced for the low probability of the pixel belonging to the cluster. Otherwise, if the membership value is greater than the threshold, then its membership value will be appropriately increased. 
If we assume that an image has three to five clusters, so the average membership value for each cluster is about from 0.3 to 0.2 . This means that if the membership value of a pixel is extremely lower than the average membership value, the pixel may be an outlier to this cluster. In our experiments, we regard the pixels whose membership value is less than 0.1 as outliers to that cluster. We transform the membership value of each pixel using the logarithmic function to make the results more accurate. Therefore, the membership function and cluster centers update of the proposed algorithm is given below:

$$
u_{i k}^{\prime}=\left\{\begin{array}{cc}
0 & u_{i k} \leq 0.1 \\
1+\frac{\ln u_{i k}}{\ln 10} & 0.1<u_{i k}<1 \\
1 & u_{i k} \geq 1
\end{array}\right.
$$

where $u_{i k}^{\prime}$ is the LF-FCM membership value and $u_{i k}$ the FCM membership value. The cluster centers can be calculated from $E q$. (5) defined as follows:

$$
c_{i}=\frac{\sum_{k=0}^{n-1} u_{i k}^{\prime m} x_{k}}{\sum_{k=0}^{n-1} u_{i k}^{\prime m}}
$$

The LF-FCM algorithm defines a transformation function, which is used to modify the membership grade from the FCM. The LF-FCM algorithm is more stabilized in our experiments, since the influence of outliers will be reduced during the update of the cluster centers, which is based on the concept of the type-2 fuzzy logic. The results of experiments show that the effectiveness of the proposed algorithm is promising.

\section{Experimental Results and Analysis}

We take some numerical examples and several UAV images for the experiments to verify the robustness of the proposed LF-FCM algorithm. The numerical example helps us to visualize how the clustering algorithms work for a comparison. Experimental results of UAV images demonstrate the superiority of the LF-FCM algorithm. For the simplicity, two clusters are used in the numerical example. In the experiments of UAV images, three clusters are used for the segmentation. In order to further examine the effectiveness of the proposed algorithm, we computed the quantitative measure of segmented UAV images. Segmentation results are evaluated using Davies-Bouldin $(D B)$ index, Relative Error Rate $\left(P_{\text {error }}\right)$ and the Relative Ultimate Measurement Accuracy $(R U M A)$.

\subsection{A Numerical Example}

To compare the clustering behaviors and results of K-Means, FCM, PCM and LF-FCM, a numerical example is tested as shown in Figure 1. There are two terminating conditions: the difference between two membership values is set to be less than 0.00001 and the maximum number of iterations is 100. Figure 1(a) presents a data set which consists of two clusters of points which are plotted with red and green colors. The members of two clusters are scattering at left-hand and right-hand sides. Please note that a dot lies in between of these two clusters, however, the point is closer to the right-hand cluster. In other words, this point should be assigned to the right-hand cluster. As we can see from Figure 1(b) to 1(e), the clustering results of the K-Means algorithm, FCM algorithm and PCM algorithm are not as robust as those of the LF-FCM algorithm. In our repeated experiments, the clustering results of the LF-FCM are always correct, but, the clustering results of the other three algorithms are not stable at all; their results are not always correct. The proposed LF-FCM algorithm performs very well, since the algorithm 
changes the membership grade of the points which makes the clustering results more accurate.

Through the logarithmic functional transformation, the LF-FCM algorithm will form a new membership function in the clustering procedure. And this enlarges the large membership and decreases the small membership for increasing their difference in order to obtain more accurate segmentation results. This transformation in the LF-FCM algorithm will contribute more precisely to the update of cluster centers. So the LF-FCM performs very well int the numerical experiments.

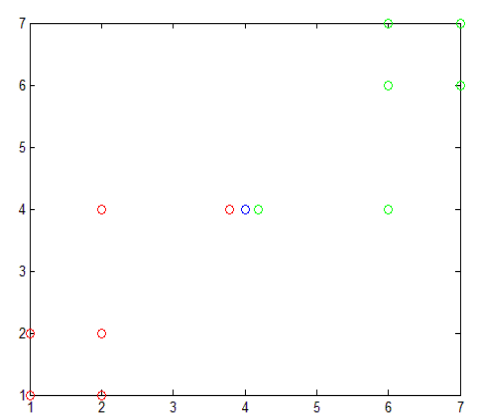

(a)
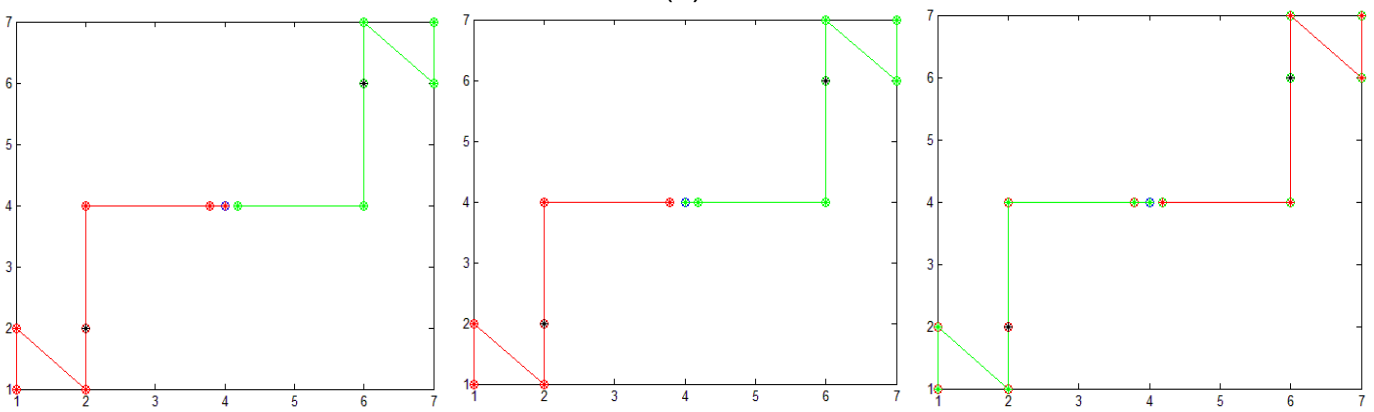

(b)
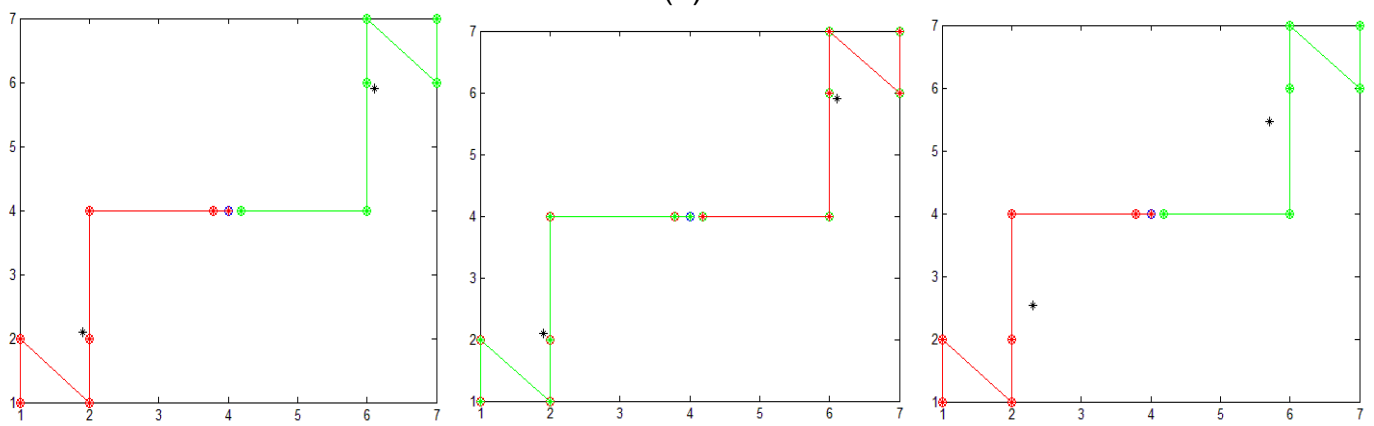

(c)
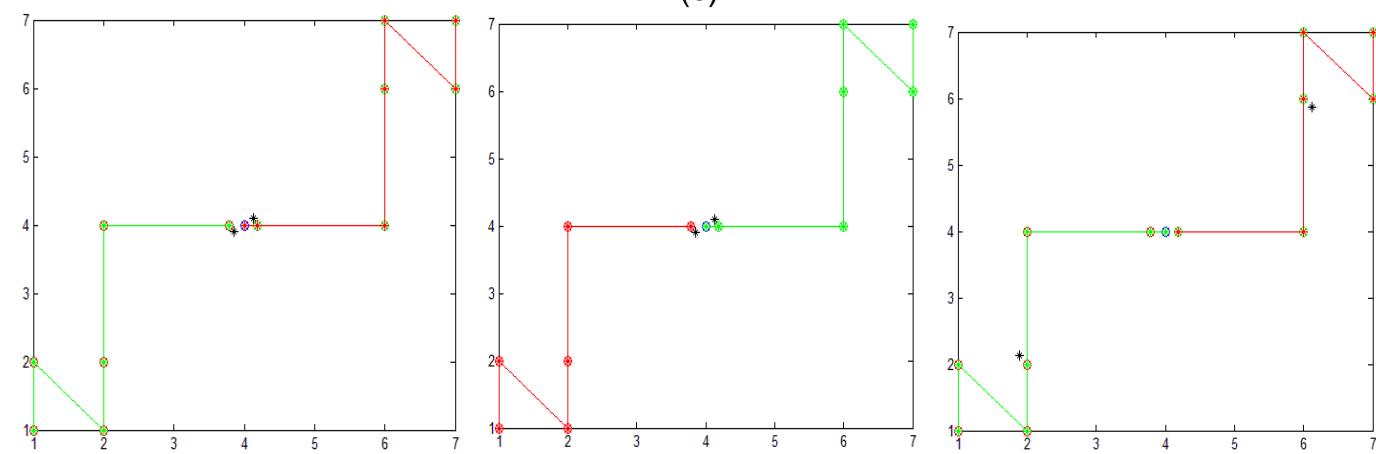

(d) 

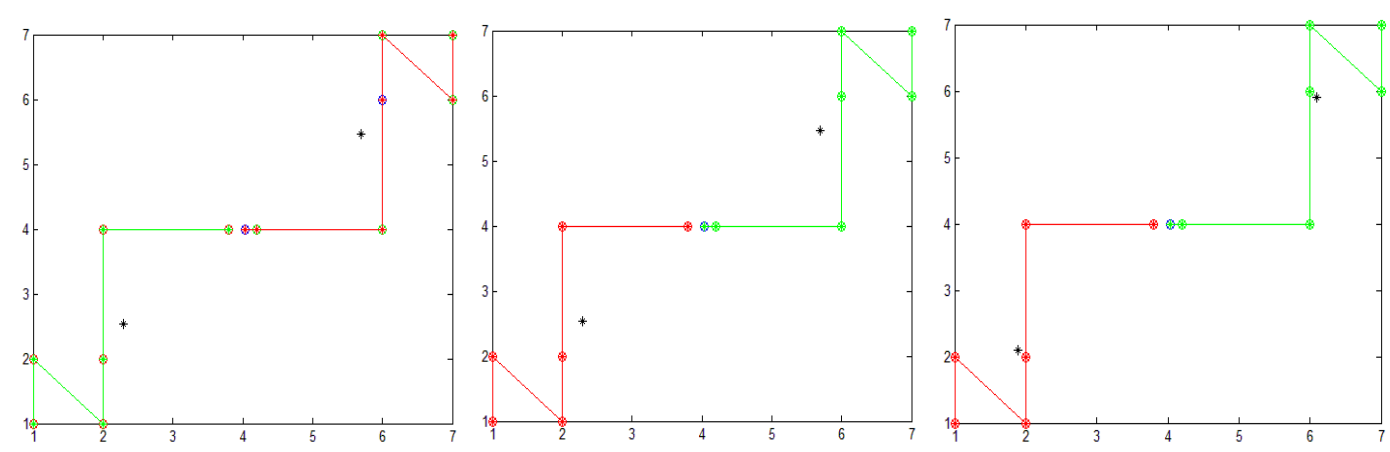

(e)

Figure 1. (a) A Data Set Consists of Two Clusters with One Cluster Marked with the Red Color on the Left-Hand Side and the Other Cluster Marked with the Green Color on the Right-Hand Side, (b) Final Clustering Results of KMeans Algorithm, (c) Final Clustering Results of FCM Algorithm, (d) Final Clustering Results of PCM Algorithm, and (e) Final Clustering Results of LFFCM Algorithm

\subsection{UAV Images Experiments}

Due to the project requirement of the Special Fund for Forest Scientific Research in Public Welfare Industry, we collected the UAV images as experimental images in JianPing Forestry Center, LiaoNing Province, China. To compare our proposed LF-FCM algorithm, we choose the K-Means algorithm, FCM algorithm and PCM algorithm for the image segmentation. We used the same number of clusters for all four algorithms to test on the UAV images, and the clustering number is three. The segmentation results are shown in Figure 2 to Figure 7.

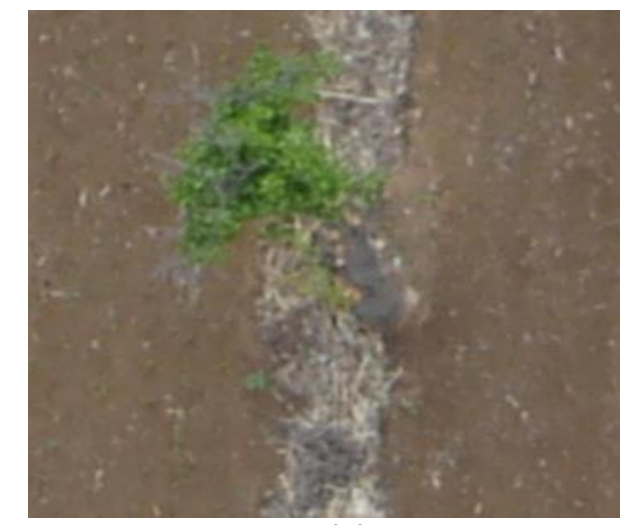

(a)

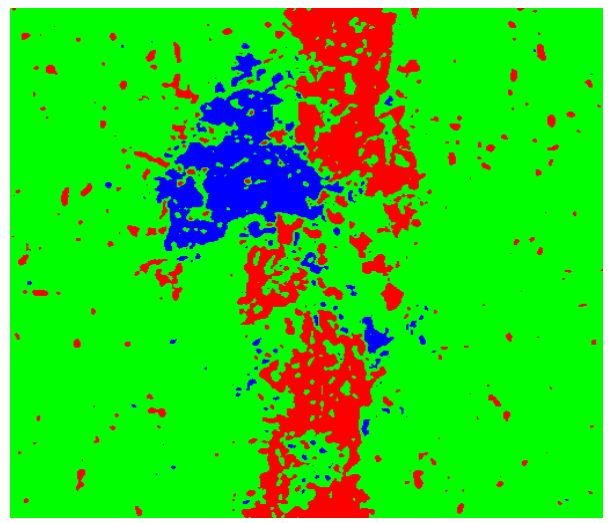

(c)

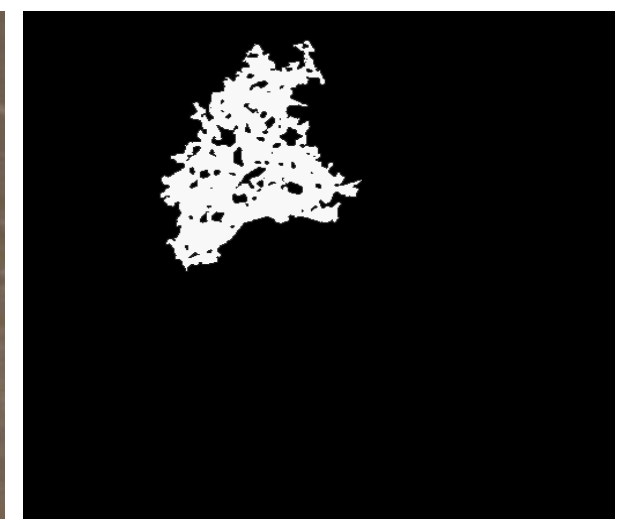

(b)

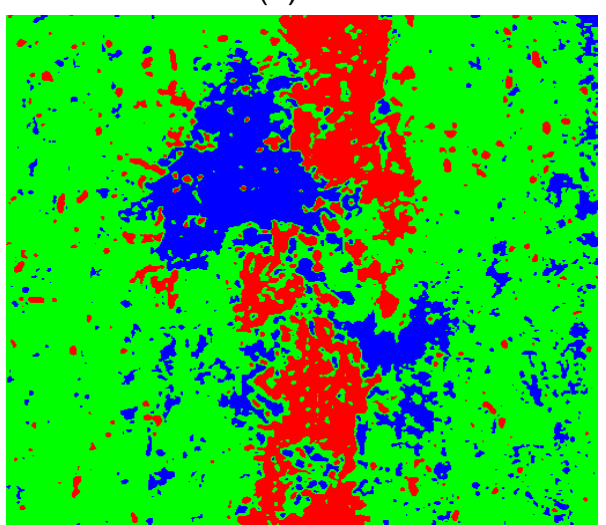

(d) 


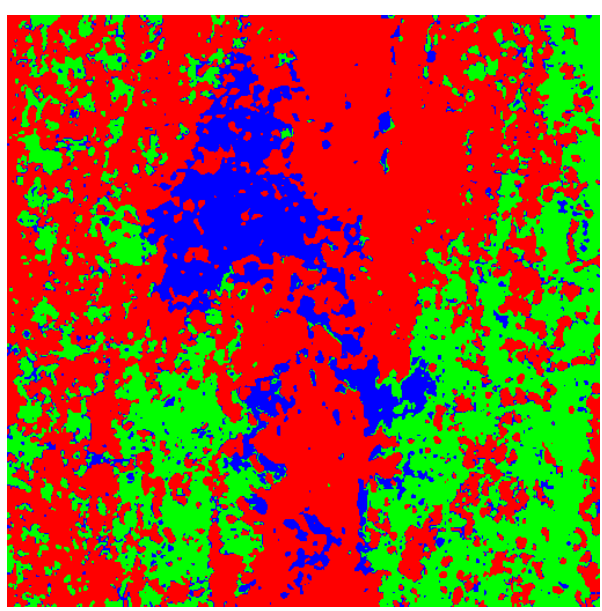

(e)

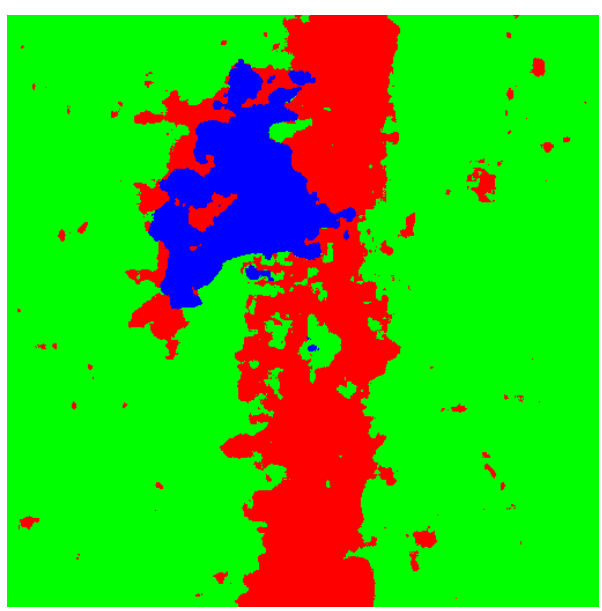

(f)

Figure 2. (a) An Original UAV Image, (Bb Result of Manual Segmentation, (c) Result of K-Means Algorithm, (d) Result of FCM Algorithm, (e) Result of PCM Algorithm, and (f) Result of the Proposed LF-FCM Algorithm

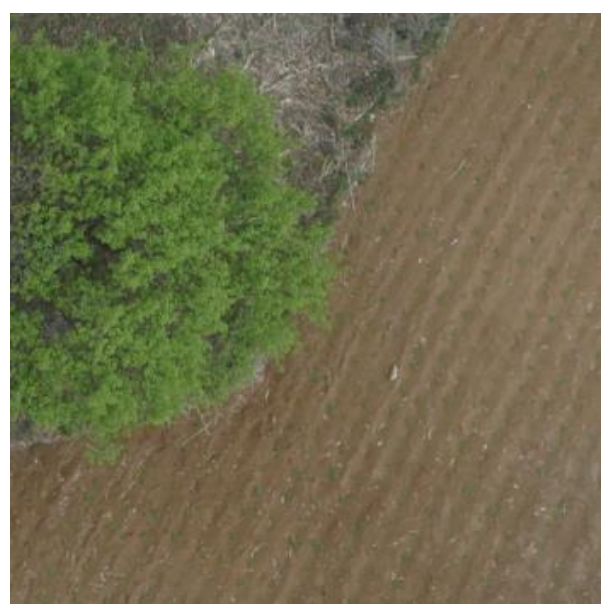

(a)

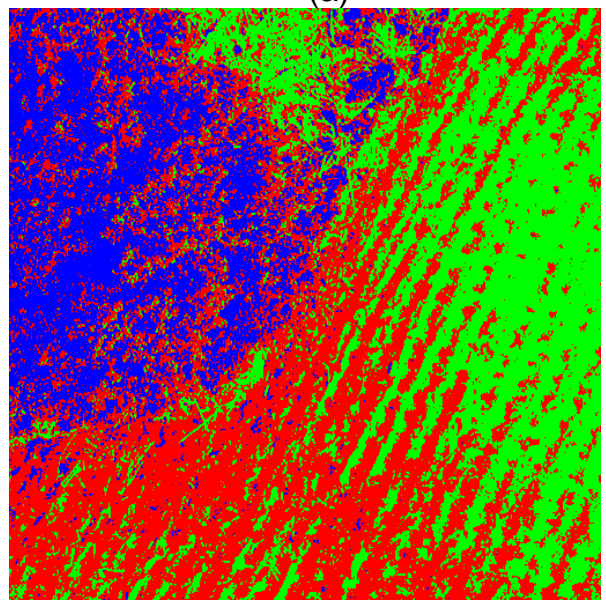

(c)

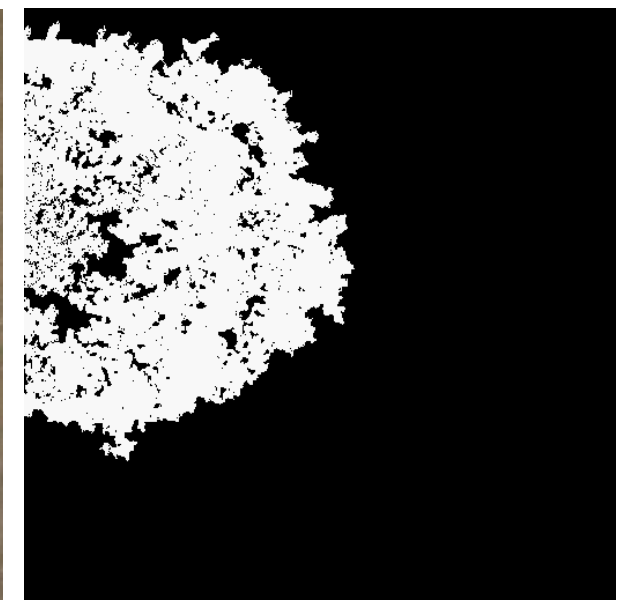

(b)

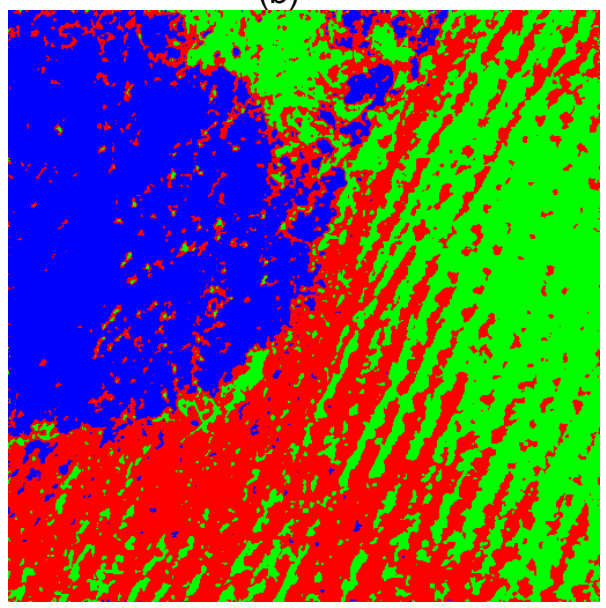

(d) 


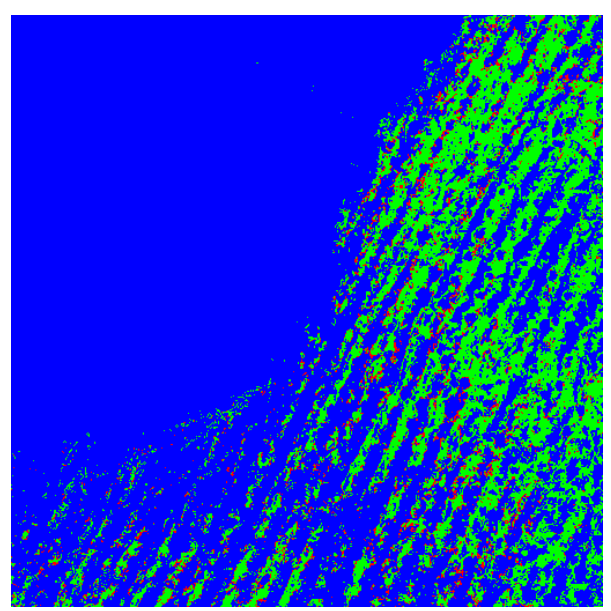

(e)

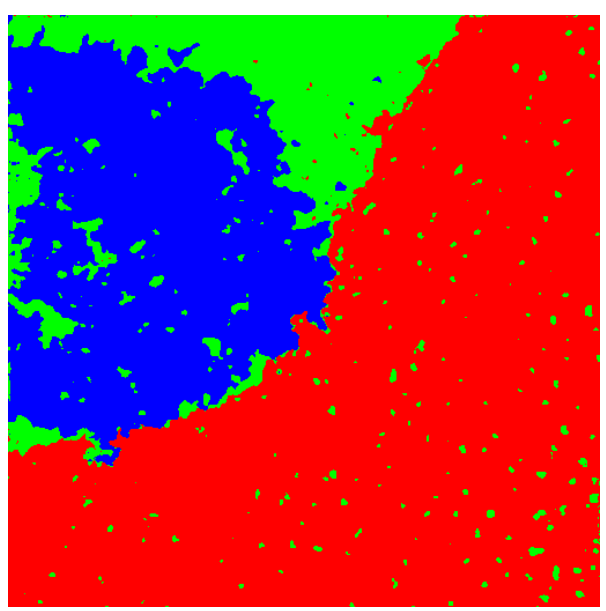

(f)

Figure 3. (a) An Original UAV Image, (b) Result of Manual Segmentation, (c) Result of K-Means Algorithm, (d) Result of FCM Algorithm, (e) Result of PCM Algorithm, and (f) Result of the Proposed LF-FCM Algorithm

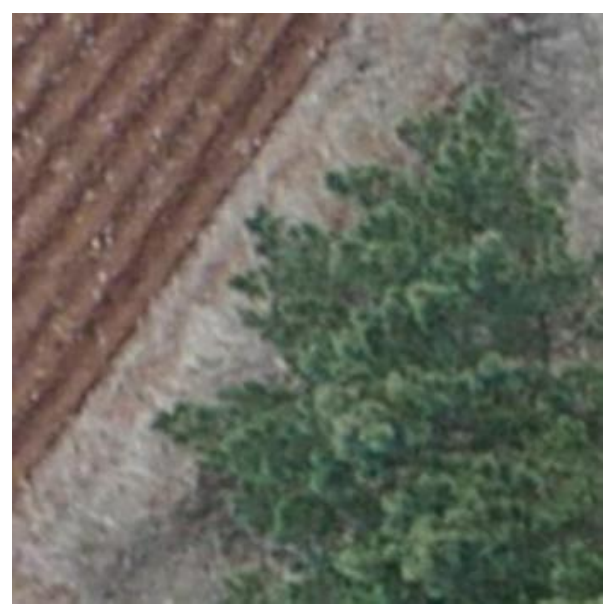

(a)

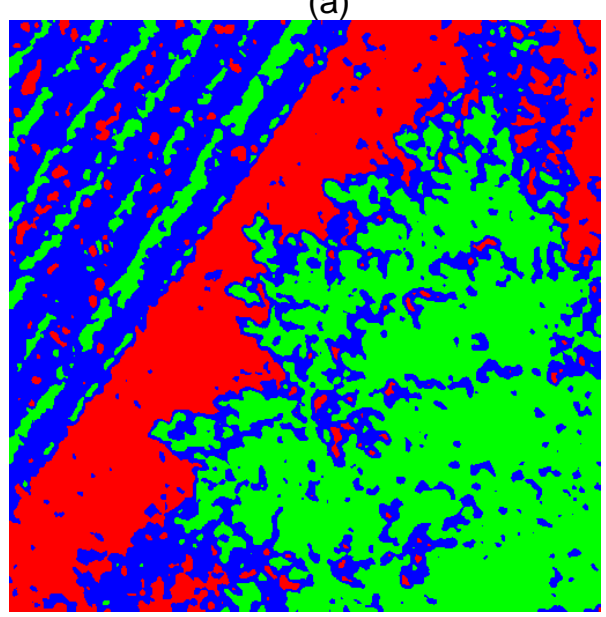

(c)

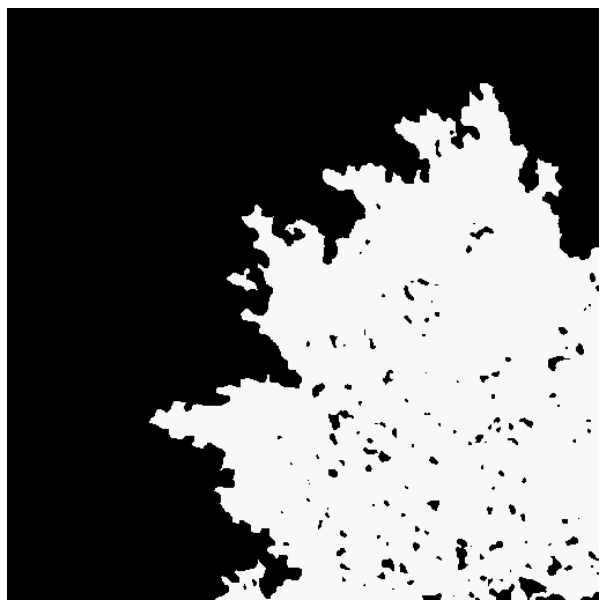

(b)

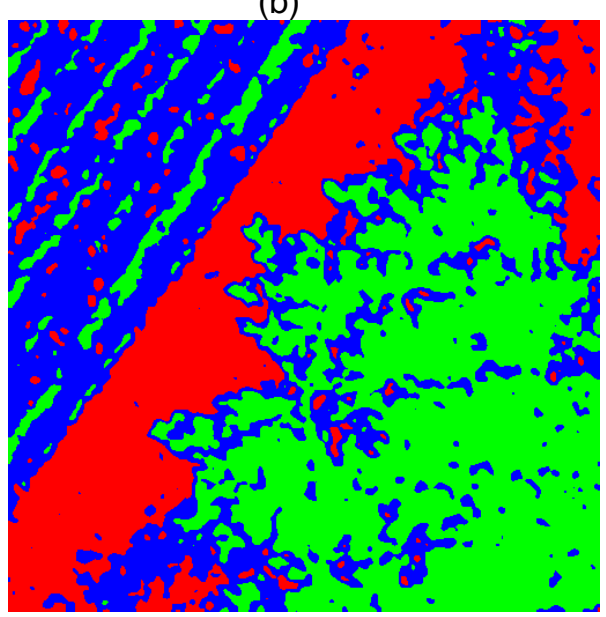

(d) 


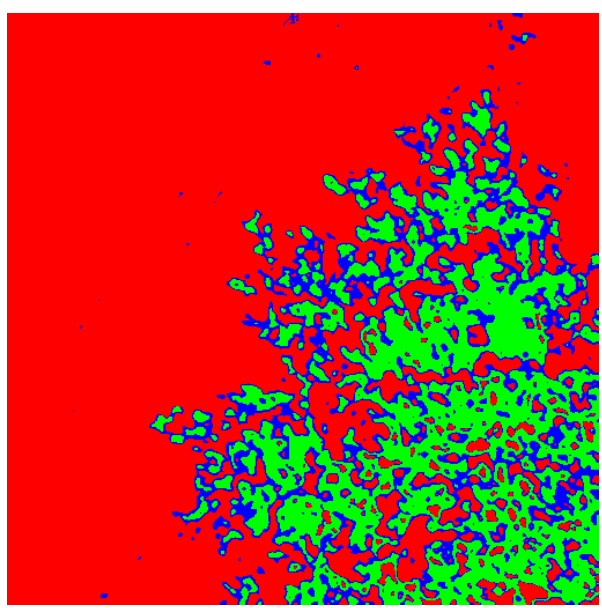

(e)

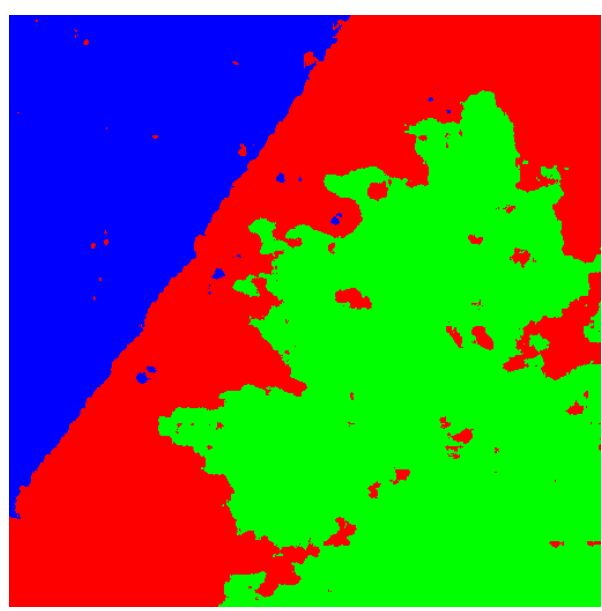

(f)

Figure 4. (a) An Original UAV Image, (b) Result of Manual Segmentation, (c) Result of K-Means Algorithm, (d) Result of FCM Algorithm, (e) Result of PCM Algorithm, and (f) Result of the Proposed LF-FCM Algorithm

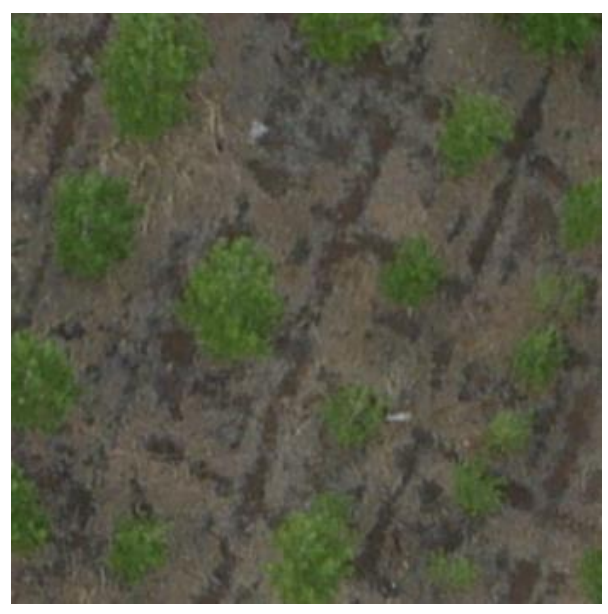

(a)

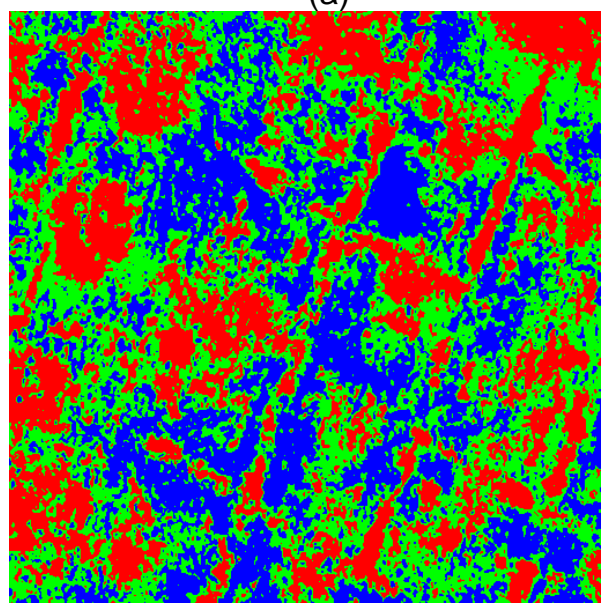

(c)

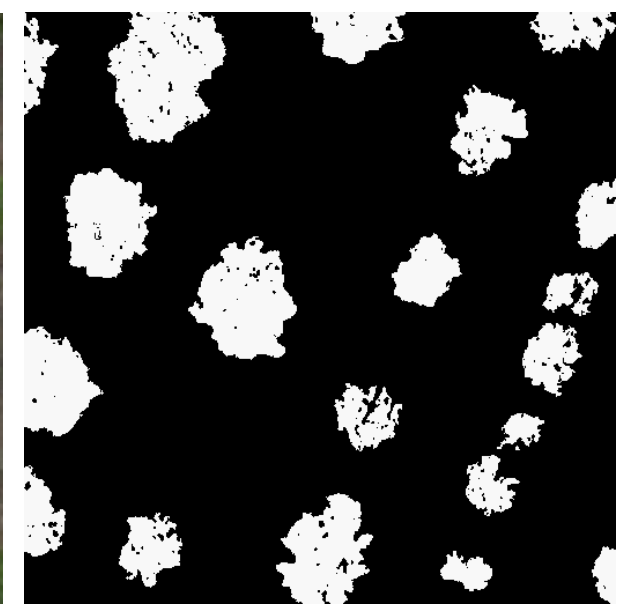

(b)

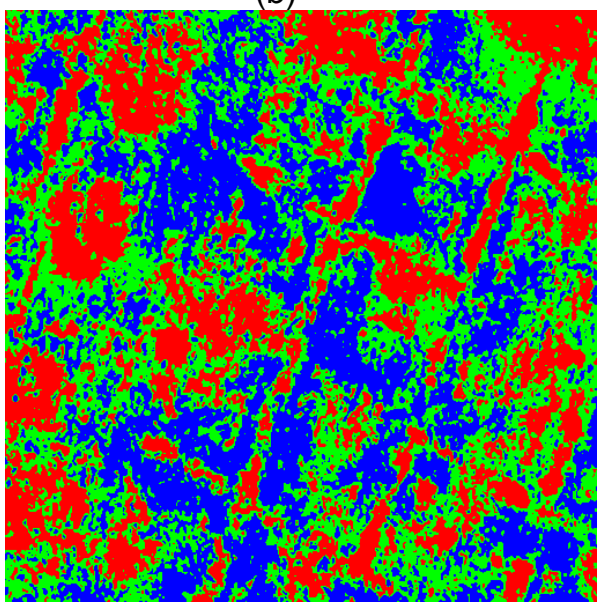

(d) 


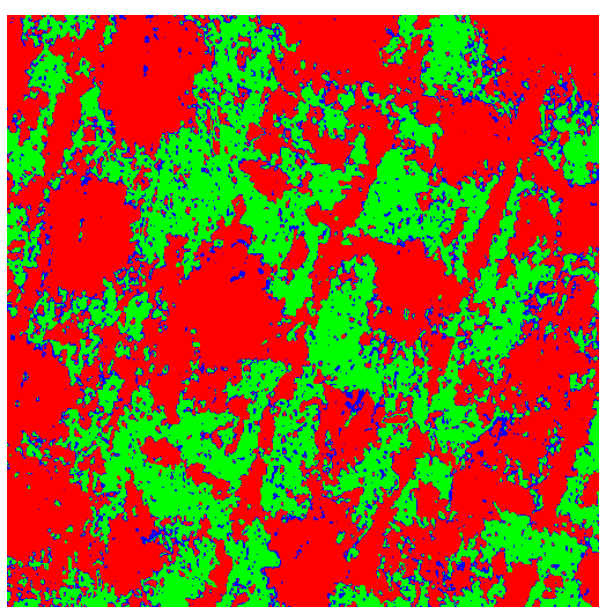

(e)

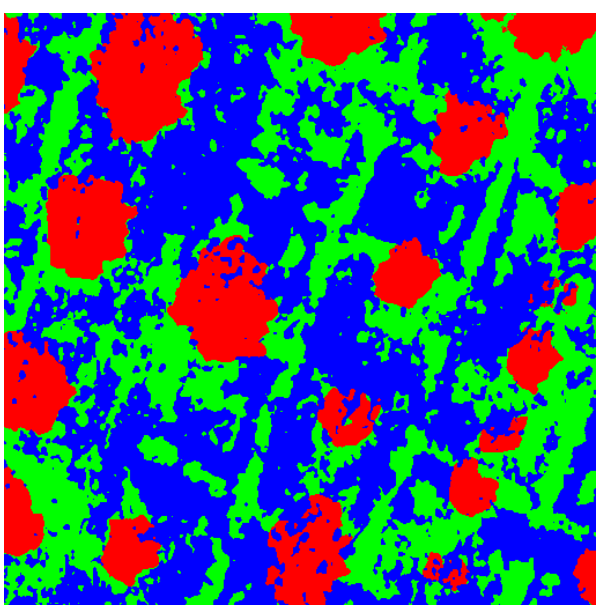

(f)

Figure 5. (a) An Original UAV Image, (b) Result of Manual Segmentation, (c) Result of K-Means Algorithm, (d) Result of FCM Algorithm, (e) Result of PCM Algorithm, and (f) Result of the Proposed LF-FCM Algorithm

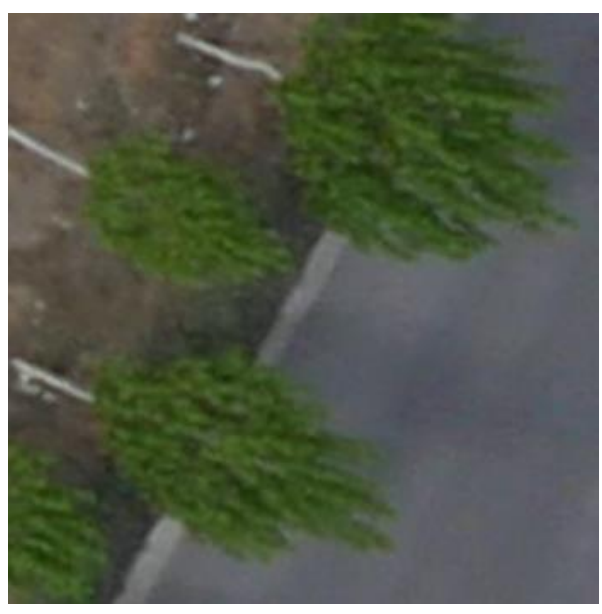

(a)

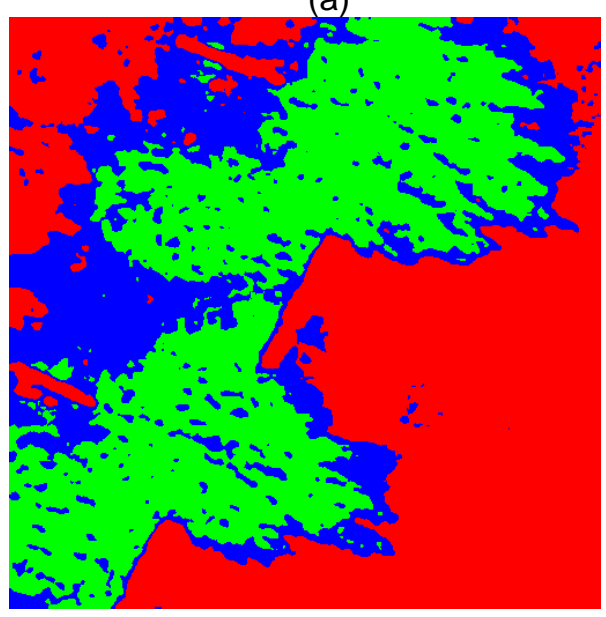

(c)

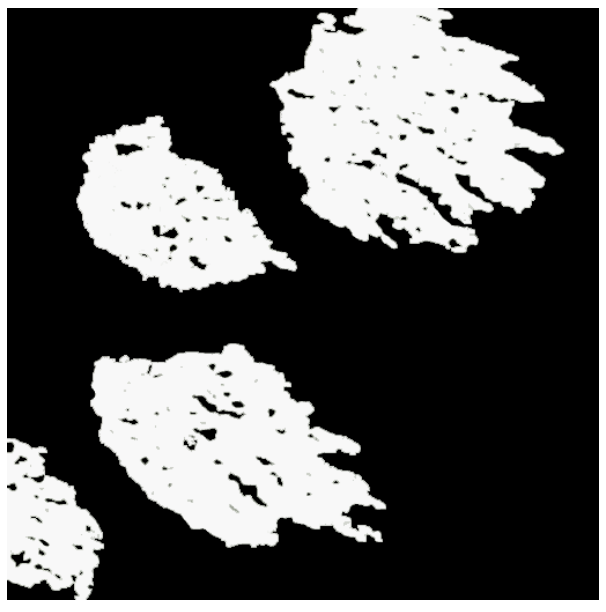

(b)

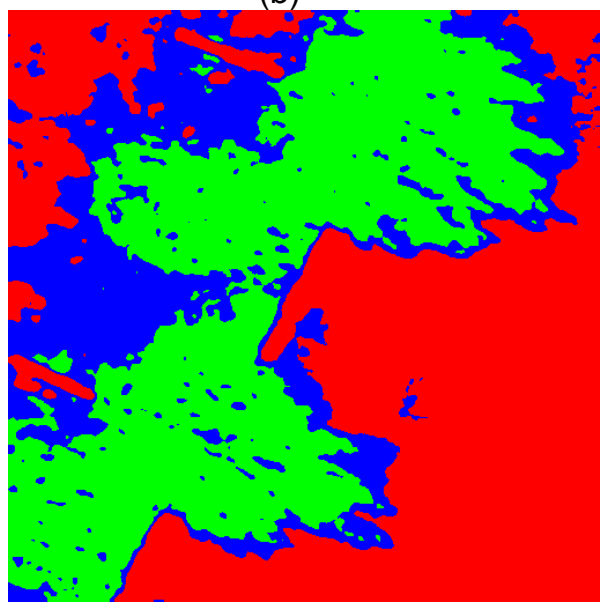

(d) 


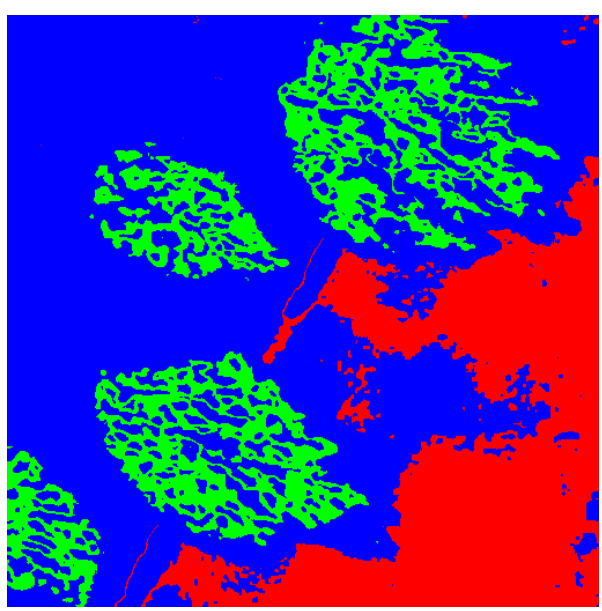

(e)

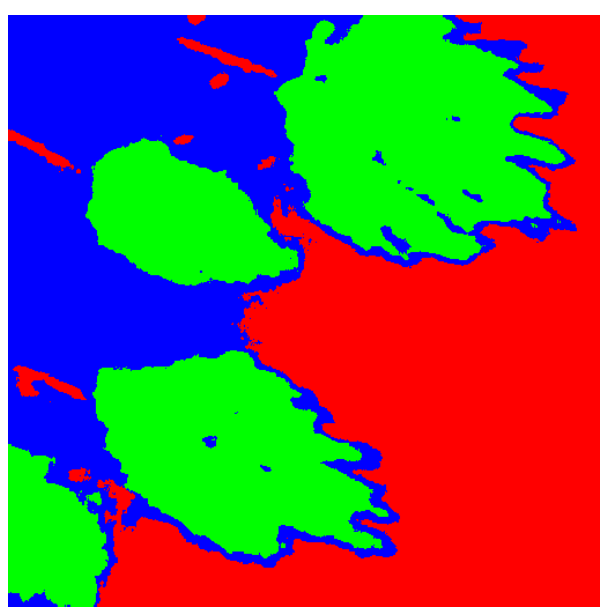

(f)

Figure 6. (a) An Original UAV Image, (b) Result of Manual Segmentation, (c) Result of K-Means Algorithm, (d) Result of FCM Algorithm, (e) Result of PCM Algorithm, and (f) Result of the Proposed LF-FCM Algorithm

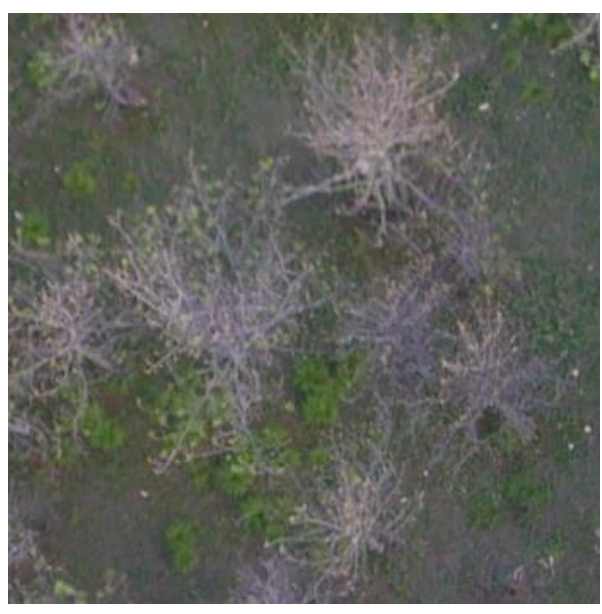

(a)

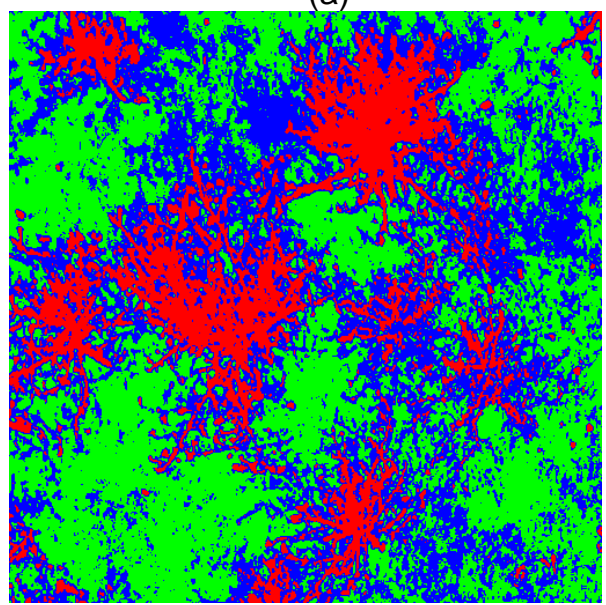

(c)

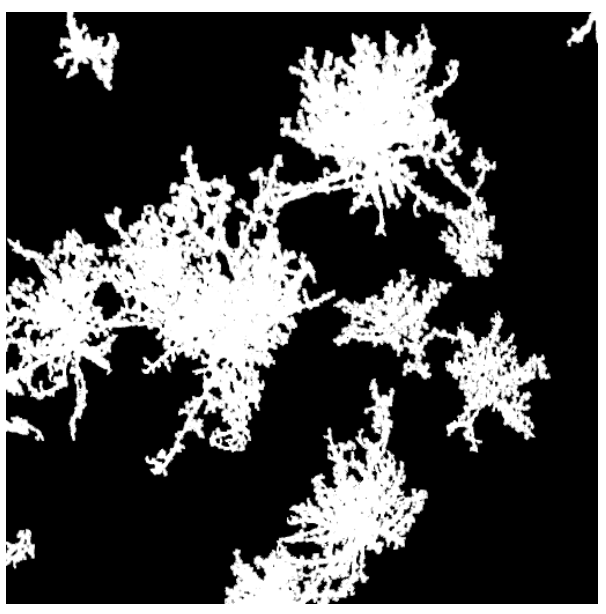

(b)

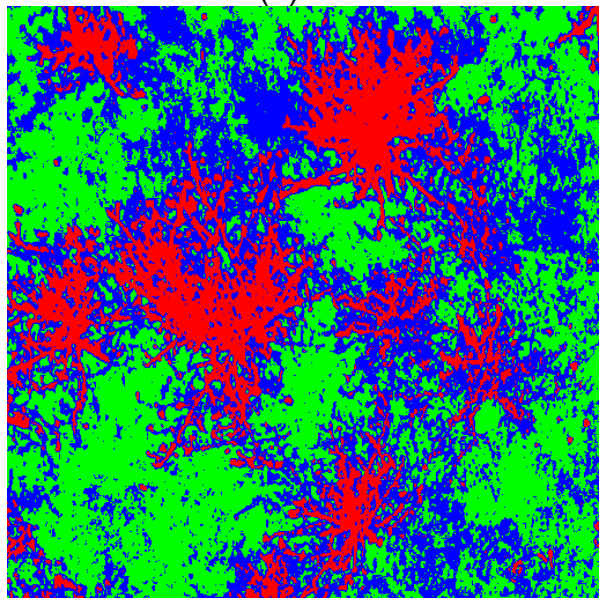

(d) 


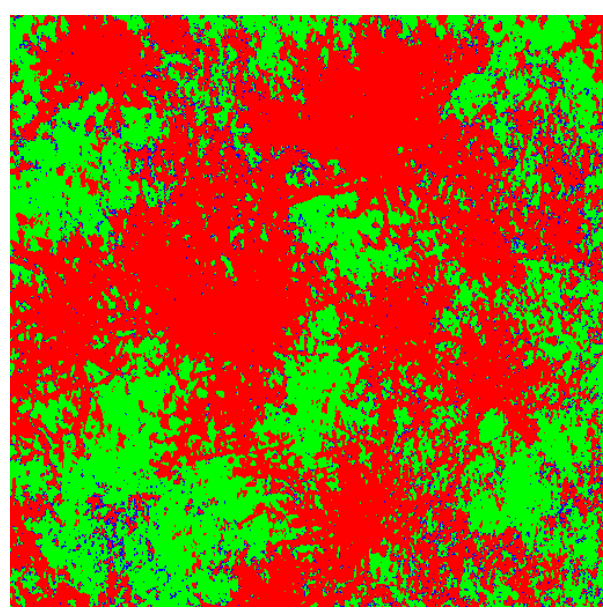

(e)

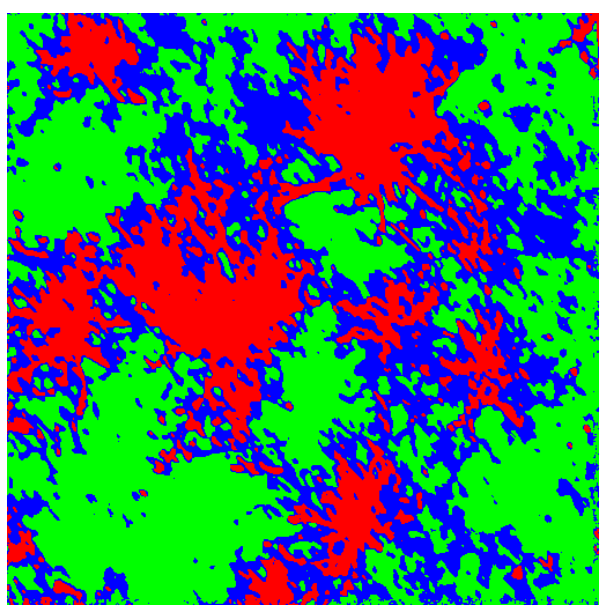

(f)

\section{Figure 7. (a) An Original UAV Image, (b) Result of Manual Segmentation, (c) Result of K-Means Algorithm, (d) Result of FCM Algorithm, (e) Result of PCM Algorithm, and (f) Result of the Proposed LF-FCM Algorithm}

In the above six natural UAV images, there exist three different regions and we focus on finding the area of the trees. The segmentation results show that the PCM algorithm has a really serious problem of over-segmentation. As we can see in Figure 3(e), the PCM algorithm cannot distinguish the tree and the sand area. Compared with the PCM algorithm, both K-Means and FCM algorithms derive the better regional connectivity. But the results of these two algorithms have many isolated points. For example, Figure 2(c) and 2(d) have many separated pixels which are segmented in the wrong clusters, because the type- 1 fuzzy set algorithms are sensitive to images with noise. However, as we can see from Figures 2 to 7, the proposed LF-FCM algorithm performs better than the above three algorithms and its clustering results approximate human visual characteristic. Particularly in Figure 5, our LF-FCM algorithm finds those trees accurately, and it differentiates the bare land and the sand land in a precise way. Because the proposed LF-FCM algorithm is based on the type-2 fuzzy logic concept, the LF-FCM algorithm has increased the difference between high membership and low membership from type-1 fuzzy set. So the LF-FCM algorithm has superior segmentation results than others.

In order to further examine the effectiveness of the proposed algorithm, we computed the quantitative measure of segmented UAV images. Segmentation results are evaluated using Davies-Bouldin $(D B)$ index, Relative Error Rate $P_{\text {error }}$ and the Relative Ultimate Measurement Accuracy (RUMA). Davies-Bouldin $(D B)$ clustering index is introduced to measure the tightness of within-class pixels and dispersion degree of inter-class pixels [16]. The best clustering results indicated that the distance of samples in the same cluster is small and the distance of samples in the different clusters are large. Therefore, the smaller $D B$ index represents the better clustering results. The $D B$ index is defined in Eq. (6):

$$
D B=\frac{1}{c} \sum_{i=1, i \neq j}^{c} \max \left(\frac{\sigma_{i}+\sigma_{j}}{d_{i, j}}\right)
$$

where $c$ is the number of clusters, $d_{i, j}$ the distance of clusters, and $\sigma_{i}$ and $\sigma_{j}$ the average distance of whole samples in clusters $i$ and $j$.

The $P_{\text {error }}$ is used to describe the rate of misclassification between the target and the background. A smaller $P_{\text {error }}$ index indicates the better segmentation result [17]. The relative rate of misclassification $P_{\text {error }}$ is defined in $E q$. (7): 


$$
P_{\text {error }}=\sum_{i=0}^{s-1} \frac{\left|N_{i}-N_{i}^{*}\right|}{N} \times 100 \%
$$

where $N_{i}$ is the number of pixels in cluster $i$ after segmentation, $N_{\mathrm{i}}^{*}$ the total actual number of pixels of cluster $i$, and $N$ the total number of pixels in the image.

The RUMA represents the performance of image segmentation. The value of RUMA is inversely proportional to the quality of segmentation, which a smaller RUMA value means a better performance for the algorithm. The definition of $R U M A$ is shown in $E q$. (8):

$$
R U M A=\frac{\left|R_{f}-S_{f}\right|}{R_{f}} \times 100 \%
$$

where $R_{\mathrm{f}}$ is the number of real pixels (ground truth) in the manual segmented region and $S_{\mathrm{f}}$ is the number of pixels in the segmented region after segmentation.

The diagrams represent more perceptual intuition and more precise comparison. The DB index, $P_{\text {error }}$ and the RUMA values of the damaged Chinese pines are shown in Figures 8,9 and 10 .

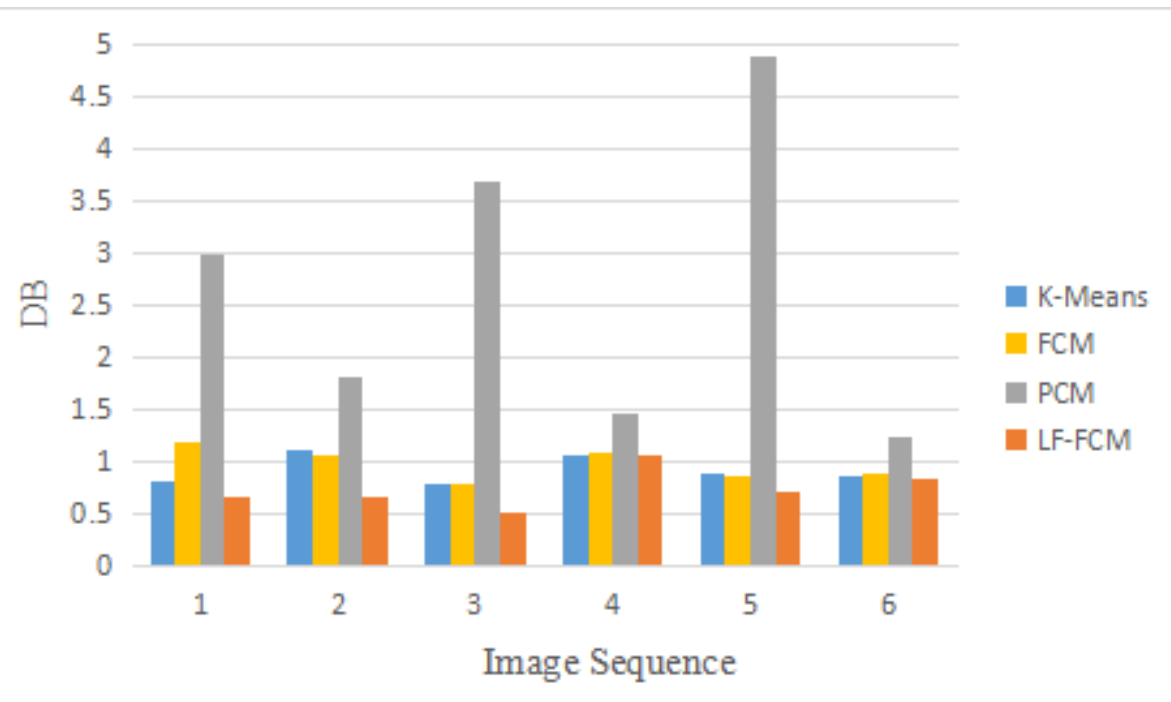

Figure 8. A Comparison of the $D B$ Indexes on All UAV Images Tested. The $X$-Axis Represents all Six Images in Our Experiments 


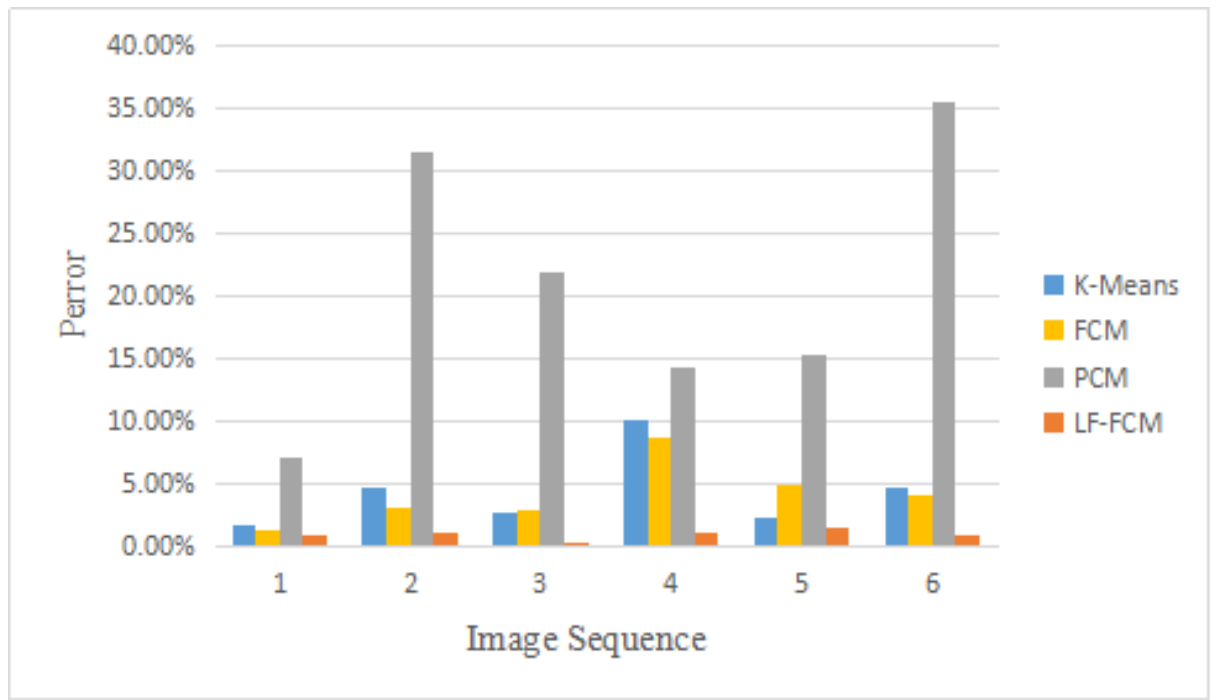

Figure 9. A Comparison of the $P_{\text {error }}$ on All UAV Images Tested. The X-Axis Represents All Six Images in Our Experiments

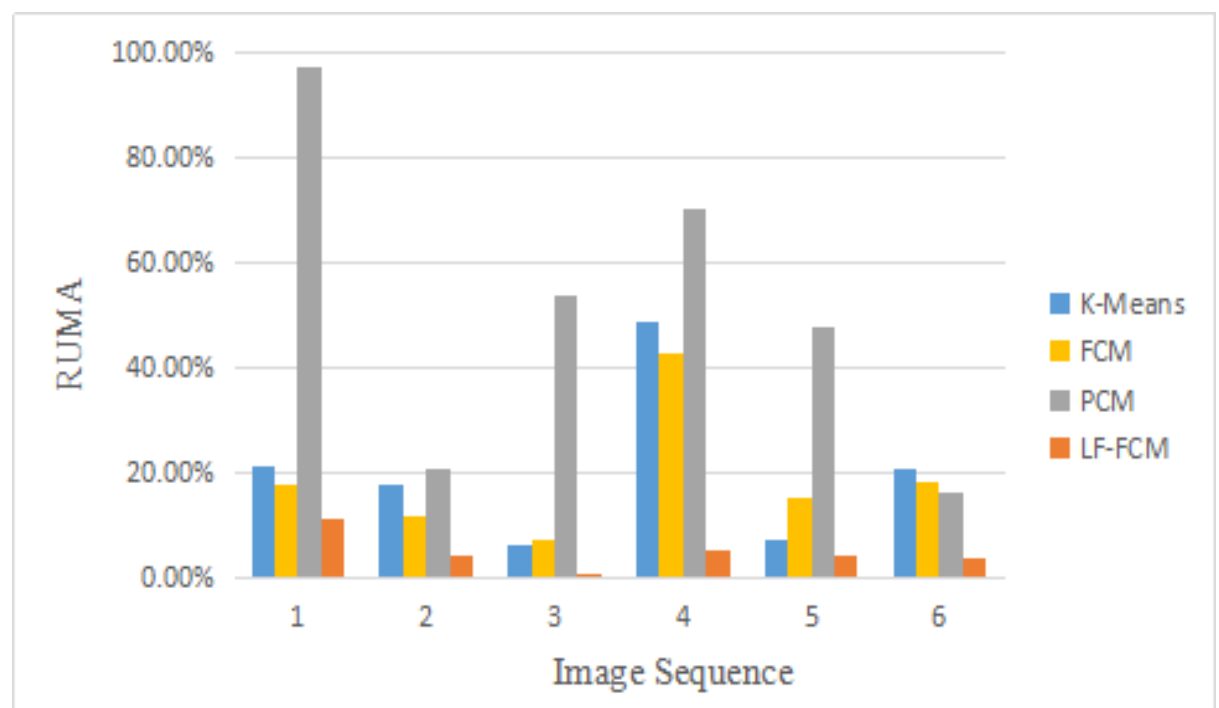

Figure 10. A Comparison of the RUMA Values on All UAV Images Tested. The X-axis Represents All Six Images in Our Experiments

From Figure 8, we can find some details of the $D B$ index from the UAV images. The $D B$ values of the LF-FCM algorithm are the lowest compared with the algorithms tested. However, the $D B$ index values of the PCM algorithm are all greater than 1.0, because all results are over-segmented. As we can see from Figure 9, we can find that the LF-FCM algorithm gives the smallest $P_{\text {error }}$. Most of the $P_{\text {error }}$ for PCM algorithm is higher than $15 \%$, some even reach $35 \%$. We can conclude that the LF-FCM algorithm performs better in the segmentation of UAV images in our project. From Figure 10, the RUMA value of PCM algorithm is higher than other three algorithms, and the proposed LF-FCM algorithm obtains the lowest RUMA value.

Figures. 8 to 10 show that the proposed LF-FCM algorithm has better clustering results than other algorithms. Experimental results show that the LF-FCM algorithm has advantages over other algorithms tested in terms of the clustering accuracy. The presented LF-FCM algorithm is effective in the UAV image segmentation in our project. 


\section{Conclusions}

In this paper we have proposed a new method by using the logarithm function with the FCM algorithm to segment the complex UAV images. We have compared four algorithms, K-Means algorithm, Fuzzy C-Means algorithm, Possibilistic C-Means algorithm and the proposed LF-FCM algorithm for a numerical example and UAV images. Both quantitative comparison and qualitative analysis of the experimental results were conducted for the comparison.

The proposed LF-FCM algorithm applied a natural logarithmic function to transform the membership value of FCM algorithm. The cluster centers which calculated by the proposed method converge to a more accurate center than cluster centers estimated by other algorithms tested. The proposed LF-FCM is superior to the other three algorithms. The LF-FCM solves the problem encountered in the UAV image segmentation in our project, and performs better than other classical algorithms. The reason is that a logarithm function based on the idea of type-2 fuzzy theory is applied to transform the membership of FCM effectively. In this study, our proposed algorithm is appropriate for the UAV images segmentation.

\section{Acknowledgments}

This work is supported by the Special Fund for Forest Scientific Research in the Public Welfare (No. 201404401) and the Fundamental Research Funds for the Central Universities (No. 2015ZCQ-XX).

\section{References}

[1] K. S. Tan and N. A. Isa, "Color Image Segmentation Using Histogram Thresholding - Fuzzy C-means Hybrid Approach", Pattern Recognition, vol. 44, no. 1, (2011), pp. 1-15.

[2] H. Yousefi-Banaem, S. Kermani, O. Sarrafzadeh, and D. Khodadad, "An Improved Spatial FCM Algorithm for Cardiac Image Segmentation”, 2013 13th Iranian Conference on IEEE, (2013), pp. 1 - 4.

[3] C. L. Feng, D. Z. Zhao and M. Huang, "Segmentation of Longitudinal Brain MR Images Using Bias Correction Embedded Fuzzy C-Means with Non-Locally Spatio-Temporal Regularization”, Journal of Visual Communication and Image Representation, vol. 38, (2016), pp. 517-529.

[4] D. Y. Tang, J. Yang, and S. Y. Huang, "Double Weighted FCM Algorithm for Color Image Segmentation", International Conference on Machine Learning and Cybernetics, vol. 3, (2012), pp. $1135-1138$.

[5] X. Y. Wang and J. Bu, "A Fast and Robust Image Segmentation Using FCM with Spatial Information”, Digital Signal Processing, vol. 20, no. 4, (2010), pp. 1173-1182.

[6] M. X. Zhou, Z. Li, X. Y. Liu and S. O. Sciences, "An Automatic Image Segmentation Algorithm Based on Improved FCM", Journal of South China University of Technology, vol. 24, no. 3, (2014), pp.1-7.

[7] A. Zhu and L. Yang, "An Improved FCM Algorithm for Ripe Fruit Image Segmentation", 2013 IEEE International Conference on IEEE, (2013) August 26-28.

[8] F. F. Guo, X. X. Wang and J. Shen, "Adaptive Fuzzy C-Means Algorithm Based on Local Noise Detecting for Image Segmentation”, IET Image Processing, vol. 10, no. 4, (2016), pp. 272-279.

[9] T. N. Long and B. H. Pham, "Approach to Image Segmentation Based on Interval Type-2 Fuzzy Subtractive Clustering", Intelligent Information and Database Systems. Springer Berlin Heidelberg, vol. 7197, no. 2, (2012), pp. 1-10.

[10] T. V. Nghiem, D. D. Nguyen and T. N. Long, "Intuitionistic Type-2 Fuzzy Set Approach to Image Thresholding”, Soft Computing and Pattern Recognition (SoCPaR), 2013 International Conference of IEEE, (2013) December 15-18.

[11] O. Assas and F. Benmedour, "Threshold Selection Based on Type-2 Fuzzy 2-Partition Entropy Approach", 2014 Second World Conference on IEEE, (2014) November 10-12.

[12] J. Shi, Y. Zhou, Y. Lei, "A Narrow Band Interval Type-2 Fuzzy Approach for Image Segmentation", Journal of Systems Architecture, vol. 64, (2015), pp. 86-99.

[13] V. N. Pham and T. N. Long, "Interval Type-2 Fuzzy Co-Clustering Algorithm", IEEE International Conference on Fuzzy Systems, (2015) August 2-5.

[14] X. Yang, C. Zhiguo and Z. Wen, "Type-2 Fuzzy Thresholding Using GLSC Histogram of Human Visual Nonlinearity Characteristics", Optics Express, vol. 19, no. 11, (2011), pp. 10656-10672.

[15] K. S. Tan, H. L. Wei and N. A. Isa, "Novel Initialization Scheme for Fuzzy C-Means Algorithm on Color Image Segmentation”, Applied Soft Computing, vol. 13, no. 4, (2013), pp. 1832-1852. 
[16] G. P. Coelho, C. C. Barbante, L. Boccato, R. F. Attux, J. R. Oliveira and F. J. Von Zuben, “Automatic Feature Selection for BCI: An Analysis Using the Davies-Bouldin Index and Extreme Learning Machines", Neural Networks, The 2012 International Joint Conference on IEEE, vol. 20, (2012), pp. 1-8.

[17] N. M. Noor, O. M. Rija, J. T. Ming, F. A. Roseli, H. Ebrahimian and R. M. Kassim, "Segmentation of the Lung Anatomy for High Resolution Computed Tomography (HRCT) Thorax Images", Advances in Visual Informatics. Springer International Publishing, vol. 8237, (2013), pp. 165-175. 